\title{
EXTRACCIÓN AUTOMÁTICA DE EDIFICIOS A PARTIR DE MODELOS DIGITALES DE SUPERFICIES: EL SISTEMA BUILDING
}

\author{
Automatic Building Extraction from Digital Surface Models: \\ the BUILDING system
}

\author{
José Luis Pérez García y Jorge Delgado García
}

Recibido 11/12/2006; aceptado el 16/03/2007

Resumen. En este trabajo se plantea una aproximación al complicado problema de la localización, extracción y representación vectorial de manera semiautomática de edificaciones situadas en zonas urbanas o suburbanas, a partir del análisis de modelos de superficie, lo suficientemente densos como para representar la escena urbana, y de imágenes aéreas orientadas de la zona de estudio.

El método propuesto utiliza como información de partida los modelos digitales de superficie generados de forma automática mediante técnicas fotogramétricas o de sistemas LIDAR con una densidad de malla elevada, así como de un modelo previo de forma geométrica de los edificios existentes en la zona. Estos modelos de superficie, serán tratados mediante operaciones de morfología matemática para la diferenciar los puntos que pertenecen al terreno propiamente dicho, de los pertenecientes a objetos situados sobre él, así como por momentos geométricos invariantes para obtener el rectángulo representativo del objeto localizado.

Una vez localizados los posibles edificios, así como los rectángulos que representan a cada uno de ellos, se procederá a refinar estos resultados trabajando sobre los fotogramas aéreos correspondientes. Mediante análisis digital de imágenes se realizará una extracción de bordes y con la ayuda de la transformada de Hough se obtendrá el rectángulo principal de la imagen. Este rectángulo junto con la altura del mismo extraída en la fase anterior, formará el prisma de base rectangular que representará al edificio.

Todas estas operaciones han sido implementadas en un sistema de desarrollo propio, el sistema BUILDING, cuyos resultados iniciales son presentados en este trabajo para poner de manifiesto las posibilidades y problemas de la metodología propuesta.

Palabras clave: Modelos 3D urbanos, Extracción automática, Análisis digital de imágenes.

Abstract. In this work we present an approximation to the complicated problem of the semiautomatic location, extraction and vector representation of buildings. This approximation is based on surface obtained from the high-density model analysis and of the oriented aerial images of a determinate area.

The proposed method uses as input information the digital surface models (obtained from photogrammetric process or LIDAR mapping data capture according to a very high density grid) and a previous geometric model of the form of the existent buildings in the zone. The surface models will be processed using mathematical morphology operators in order to classify ground points and non-ground points. Furthermore, the regions that have been classified as non-ground regions will be processed using invariant geometric moments in order to obtain the representative rectangle of localized object.

Departamento de Ingeniería Cartográfica, Geodésica y Fotogrametría, Universidad de Jaén.

Campus de las Lagunillas, Edif. A-3, 23071 Jaén (España).

Teléfono: (+34) 953-213353 (+34) 953212468

Correo electrónico: jlperez@ujaen.es, jdelgado@ujaen.es 
Once the possible buildings have been detected, their representative rectangles are defined. The results will be refined using the complementary information provided by aerial photogrammetric imagery. Using digital image analysis procedures it is possible to extract the main borders presented in the images and applying the Hough's transform the principal rectangle will be obtained. This rectangle combined with the heights obtained from the DSM in the same location, constitute the rectangular base prism that represent to the building.

All these operations have been implemented in an own developed system, whose preliminary results are presented in this works in order to establish the capabilities and problems of the methodology.

Key Words: City 3D Models, Automatic extraction, Digital image analysis.

\section{INTRODUCCIÓN}

En la actualidad más de la mitad de la población mundial habita zonas urbanas o suburbanas, y el desarrollo que se ha producido en ellas en estos últimos tiempos ha sido enorme. Esto lleva a que cada día sea más necesario el contar con una cartografía precisa y actualizada de dichas zonas, con vistas a la planificación y gestión de los servicios prestados a sus habitantes. Una de las características de dicha cartografía es la necesidad de proporcionar la información necesaria para poder obtener a partir de ella una descripción tridimensional de las ciudades, aspecto que aun permanece pendiente de resolver. Esta cartografía tridimensional es fundamental para diversas aplicaciones como, por ejemplo, diseño de redes de transmisión y telefonía móvil, estudios de impacto visual, de ruido o polución, a otras como estudios de simulación para predicción de inundaciones y obtención de ortofotografías verdaderas en zonas urbanas, etc.

La generación de cartografía en zonas urbanas es una tarea laboriosa que en los últimos años ha experimentado importantes avances que simplifican y agilizan su trabajo. Así se pueden mencionar dentro de estos avances la utilización de las técnicas de Fotogrametría Digital para la automatización del proceso de generación de cartografía a partir de fotografías aéreas que ha permitido la automatización parcial del mismo. Desgraciadamente, dicha automatización aún no es completa, así por ejemplo, tareas como la restitución (o de acuerdo a la necesidad concreta, la extracción automática de los volúmenes ocupados por los edificios) son tareas que aún no están automatizadas en los sistemas comerciales, contando tan sólo con algunas herramientas de asistencia para facilitar el trabajo.

Evidentemente, la extracción automática de edificios no es un problema sencillo de resolver debido a la gran cantidad de información que es interpretada por el operador. Dicha problemática queda reflejada en diversos artículos (Lin y otros, 1995; Baltsavias y otros, 1995; Weidner y otros, 1995; Lin y otros, 1998; Gerke y otros (2001) y Vosselman (2002), entre otros) poniendo de manifiesto el interés de la cuestión en el ámbito internacional.

\subsection{Objetivo}

El objetivo básico de este trabajo es plantear una metodología sencilla para la extracción automática de edificios. Dicha metodología se basa en utilización de modelos de tipo prismático, que permitirá la extracción del prisma rectangular más característico del edificio desde el punto de vista geométrico. Esta metodología ha sido implementada en un programa informático de desarrollo propio y necesita como información de partida el modelo digital de superficies de la zona de estudio y las imágenes aéreas de la zona con sus parámetros de orientación externa.

\subsection{Solución adoptada}

La solución adoptada utiliza como información de partida un modelo digital de superficies (MDS) que puede ser obtenido a partir de correlación fotogramétrica o medido mediante un sensor LIDAR. El proceso se inicia con un tratamiento global del mismo a objeto de detectar las zonas con existencia de edificaciones. Una vez localizadas, éstas son separadas de forma que se pasa a trabajar de una forma local e individualizada con cada una de las regiones extraídas, lo que facilita de una forma importante la posterior etapa de edición de los resultados mediante el análisis de las zonas de imagen aérea correspondientes a las regiones localizadas.

La representación final de cada uno de los edificios localizados va ser el prisma de base rectangular que mejor represente al mismo. Por lo tanto, esta metodología va a obtener resultados satisfactorios con edificios que se ajusten al modelo geométrico planteado (es decir, edificios con una base rectangular). Otro factor a tener en cuenta es que la localización se realizará de manera individual para cada una de las edificaciones, motivo por el cual entre las mismas deberá existir una determinada distancia, en el caso de encontrarse unidas la representación obtenida será la del conjunto de edificaciones.

En este trabajo se muestran ejemplos de aplicación de la metodología a zonas con diferentes características, tanto de las condiciones de la toma de las fotografías (diferentes cá- 
maras y escalas de vuelo) como de la morfología de las edificaciones (densidad de edificios, altura y superficie de los mismos, etc.).

\section{METODOLOGÍA}

\subsection{Estrategia general}

La metodología está compuesta de dos etapas básicas (localización de los edificios y extracción de los mismos), junto con una etapa previa de preparación inicial de los datos. El esquema general del proceso se muestra en la figura 1.

La etapa previa incluye la selección de las imágenes a emplear, la información de la calibración de la cámara o cá- maras utilizadas y del MDS cuyo formato inicial debe ser un fichero XYZ en formato ASCII. Dentro de esta etapa inicial se procede a la orientación interna de las imágenes utilizando procedimientos automáticos de localización y medida de las marcas fiduciales y la conversión del MDS de su formato original en formato ASCII a un formato binario para optimizar el espacio de almacenamiento y las posteriores operaciones que es necesario realizar a partir del mismo.

En el caso de disponer de datos brutos LIDAR, éstos previamente deben ser tratados para obtener una malla regular de espaciado adecuado (de acuerdo con la densidad de los datos y la escena a representar). En este caso si se dispone como información complementaria de la intensidad del eco, ésta puede ser utilizada posteriormente junto con los pro-

FigURA 1.

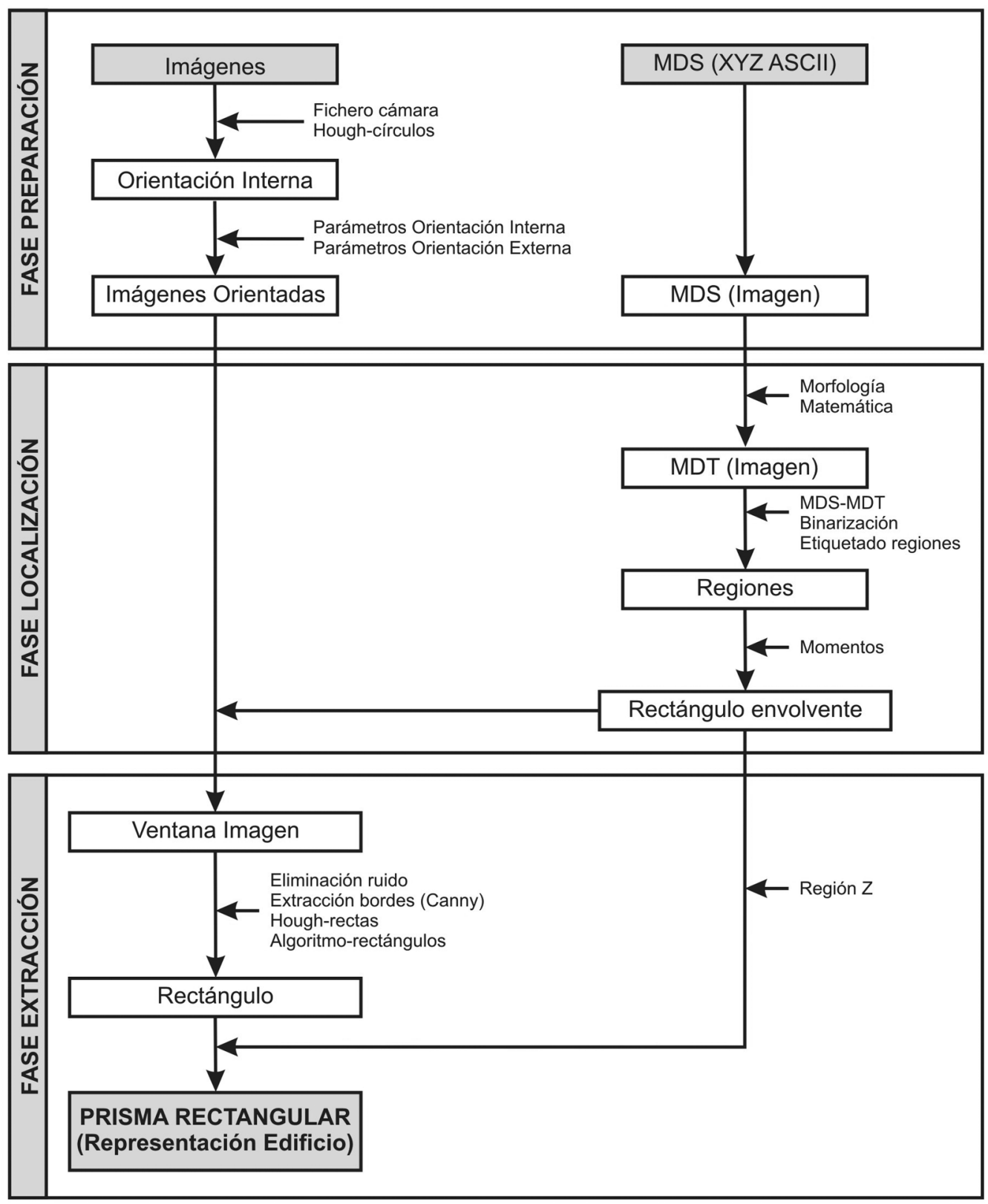


pios datos de elevación para la clasificación de los objetos en zonas de vegetación o zonas edificadas.

La primera etapa o de localización comienza con la selección de la zona a estudiar dentro de la imagen total del MDS. Esta imagen, teniendo en cuenta una serie de variables introducidas por el usuario, relacionadas con las características de los edificios que nos vamos a encontrar (superficies mínima y máxima, altura mínima, etc.) es tratada mediante morfología matemática para inicialmente y a partir del modelo original, obtener un modelo digital del terreno (MDT), que es el resultado de eliminar del MDS las edificaciones y el resto de objetos situados sobre el terreno. Posteriormente, la diferencia de ambos modelos MDS y MDT proporciona un nuevo modelo al que denominaremos modelo digital de objetos (MDO) que contiene exclusivamente los objetos sobre el terreno, a partir del cual se obtienen las regiones que representan posibles edificios. Por último, estas regiones son tratadas mediante descriptores de formas como son los momentos geométricos invariantes (Gerke y otros, 2001) obteniendo así para cada región el centro de masas, el rectángulo envolvente y el mínimo-encuadrante, así como la cota de la región.

\subsubsection{Fase Previa o de Preparación}

Esta fase consiste en preparar la información de partida para que sea posible la extracción automática propiamente dicha. La información consiste en las imágenes -y el certificado de la cámara correspondiente-, la posición y orientación de la cámara en el momento de la toma y el modelo digital de superficies con un estructura de malla regular y almacenado en formato ASCII.

Antes de comenzar con la extracción es necesario realizar la orientación interna de las imágenes. El sistema desarrollado incorpora un módulo de orientación interna automática, con detección y medida autómatica de las marcas fiduciales, permitiendo igualmente la medida de forma automática para la edición/revisión de las marcas medidas de forma automática en los casos en los que la medida no sea completamente satisfactoria.

Una vez que se han introducido en el sistema del modelo digital de superficie (convertido a formato binario imagen) y de los parámetros de orientación de la imagen (interna y externa) ya es posible iniciar el proceso en sí de la extracción de los edificios al ser posible relacionar los sistemas de coordenadas imagen y terreno.

\subsubsection{Fase de Localización}

Una vez seleccionada la zona de trabajo se extrae dicha región del modelo digital correspondiente y es necesario posible proporcionar las variables que se emplean en la localización automática. Estas variables son: superficie mínima y máxima esperada para los edificios (se utiliza para la depu- ración de las regiones extraídas), altura mínima esperada (es el valor inicial de umbralización para la binarización del MDS) y distancia de apertura (debe ser superior a la mitad del lado mayor del edificio mayor esperado). Evidentemente, la obtención de estos valores parte de un cierto conocimiento de la escena, o bien de un análisis premilinar de las caracteristicas de los edificios a extraer.

Una vez que se han asignado los valores a las diferentes variables, se procede al cálculo y localización de las regiones. Para lo cual se siguen las siguientes fases:

1. Aplicación de un proceso de apertura morfológica (González y Woods, 1996). A partir de la distancia de apertura introducida por el usuario, es posible obtener una aproximación de la superficie del terreno (MDT).

Para que la apertura no esté influenciada por la orientación de los edificios se emplea un elemento estructural de forma circular, en lugar de una ventana rectangular. En este proceso, la determinación del tamaño de apertura es fundamental y debe ser escogida en función del tamaño de los objetos a extraer y de la morfología del terreno de la zona. La elección de una distancia excesivamente pequeña la extracción será incorrecta quedando objetos que no serán extraídos o bien que serán extraídos de forma errónea, por otro lado, las distancias muy grandes eliminarán objetos pequeños y generará la extracción errónea de zonas del terreno elevadas con respecto a su entorno. Estos problemas pueden ser minimizados con el empleo de filtros más sofisticados y complejos como el slope adaptative filter (Sithole, 2001) o el progressive morphological filter (Zhang y otros, 2003) que permiten trabajar adaptándose a diferentes pendientes así como a diferentes tamaños de objetos dentro de la misma escena.

Como resultado de esta fase se obtienen dos modelos diferentes: un modelo digital del terreno (MDT) obtenido de la eliminación de los objetos existentes sobre el MDS y el modelo digital de los objetos (MDO) que contiene sólo los objetos situados sobre el terreno (Figuras 2 y 3 ).

2. En el caso de disponer de datos de intensidad LIDAR es posible realizar una preclasificación de los objetos en vegetación y edificios. Para ello se tendrá en cuenta datos de intensidad (muy baja en zonas de vegetación) y de curvatura (baja en los tejados de los edificios). De esta manera, podremos centrarnos para la extracción de los edificios solo en las regiones con alta probabilidad de ser edificaciones (figura 4).

3. El modelo digital de los objetos es sometido a un doble proceso de binarización a objeto de realizar la localización de los edificios. La primera binarización se 
FigurA 2.
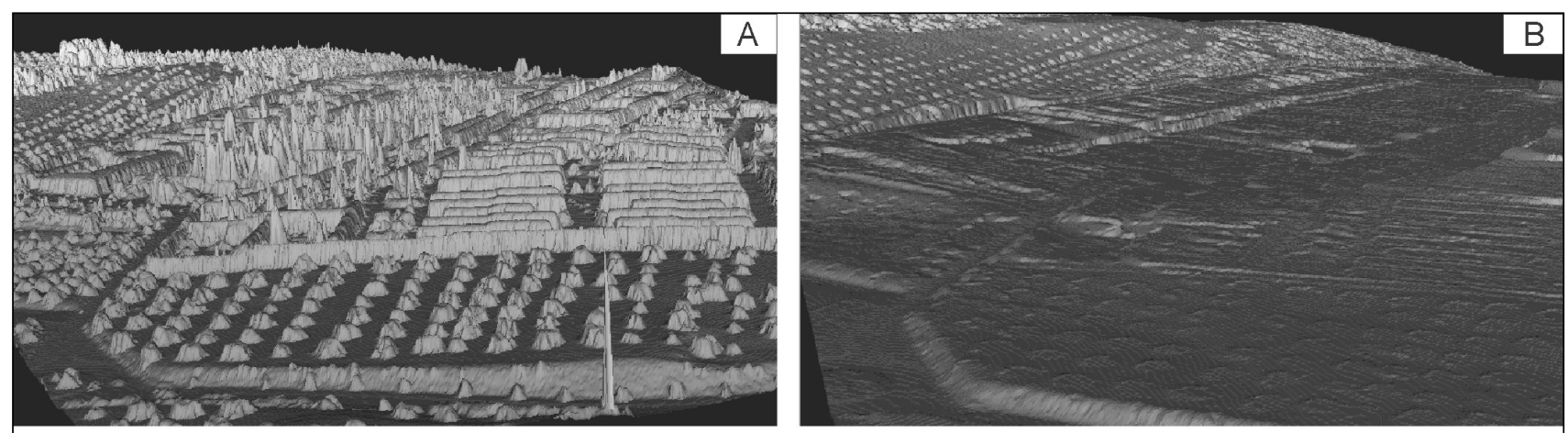

C

establece en función de la altura mínima de edificio esperada mientras que la segunda se plantea a partir de una pendiente máxima a fin de detectar los bordes de los edificios.

4. Tras esta operación, se realiza una agrupación de píxeles pertenecientes a la misma región y se procede al etiquetado de las regiones. Las regiones obtenidas en esta fase se depuran teniendo en cuenta los datos de superficie máxima y mínima introducidos por el usuario introduciendo como criterio adicional el que no estén situadas en el margen del modelo para evitar la aparición de edificios no completos.

5. Obtención de las características de las regiones mediante el uso de momentos geométricos inerciales

FigURA 3.

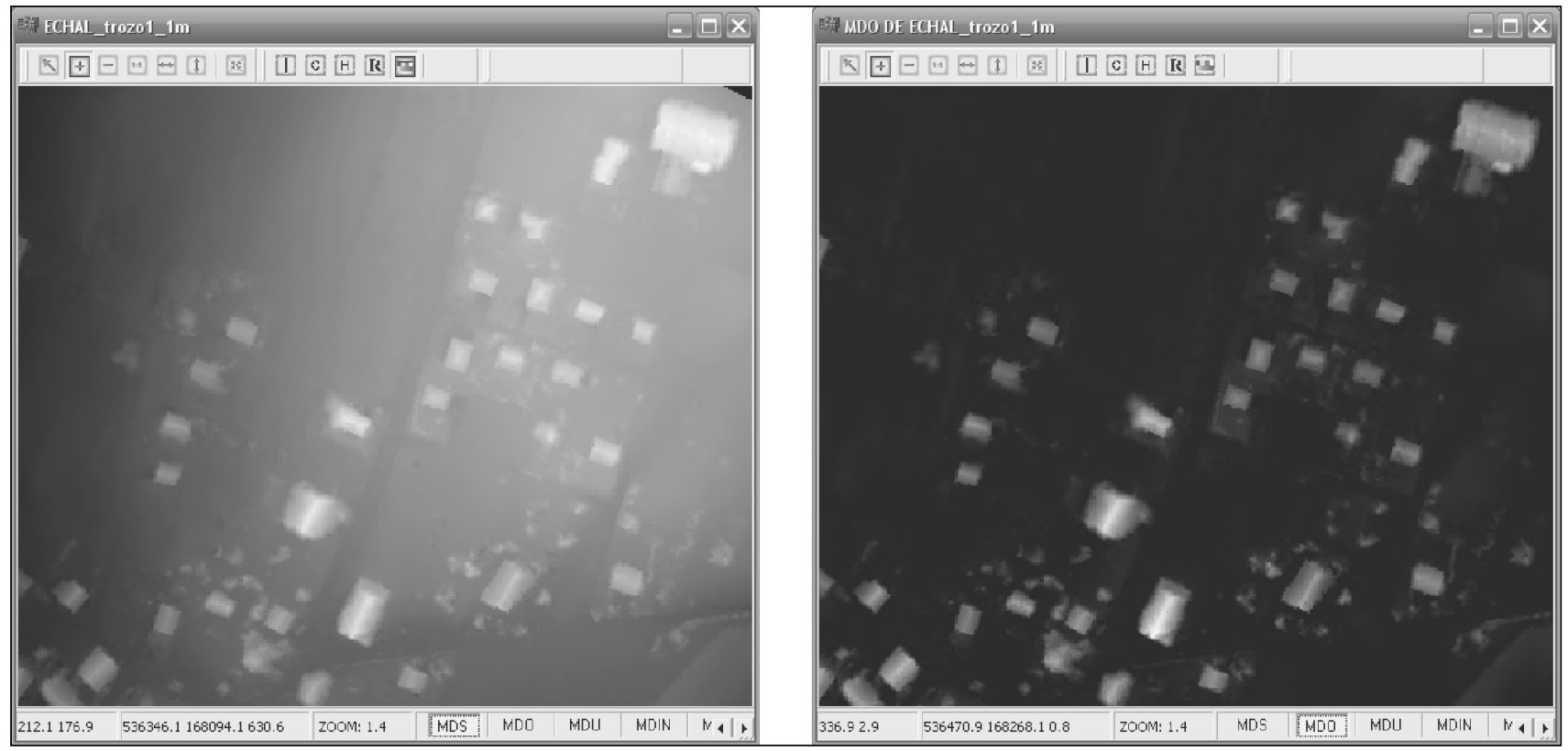


Figura 4.
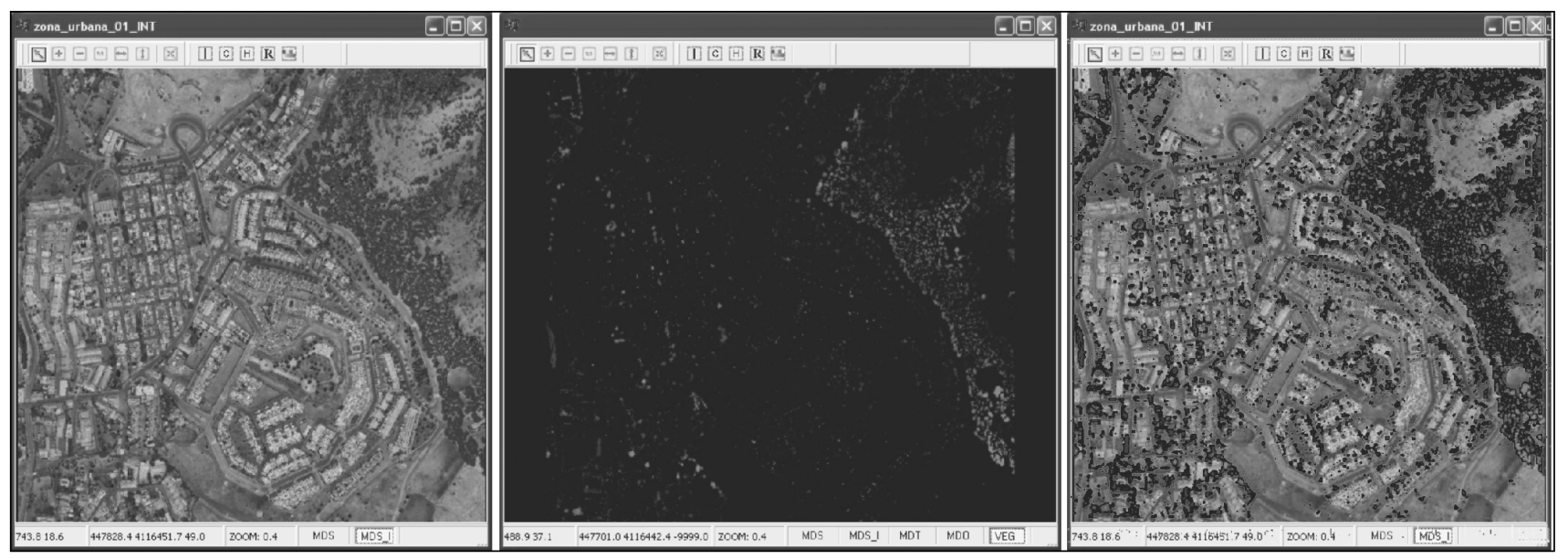

(Gerke y otros, 2001). En esta fase se obtiene para cada región información básica como la superficie, el centro de masas, la altura media de la región, el rectángulo mínimo-encuadrante y el rectángulo envolvente. Para lo que se utilizará la formulación siguiente:

- Centro de masas:

$$
\bar{x}=\frac{1}{N} \sum_{x} \sum_{y} x=m_{1,0} \quad \bar{y}=\frac{1}{N} \sum_{x} \sum_{y} y=m_{1,0}
$$

- Orientación: Se define como el ángulo del eje correspondiente al mismo momento de inercia, y se obtiene minimizando dicha función.

$$
\begin{aligned}
& I(\theta)=\sum_{x} \sum_{y} d^{2} \min (x, y)= \\
& =\sum_{x} \sum_{y}[(y-\bar{y}) \cos \theta-(x-\bar{x}) \operatorname{sen} \theta]^{2} \\
& \theta=\frac{1}{2} \arctan \frac{2 \mu_{1,1}}{\mu_{2,0}-\mu_{0,2}}
\end{aligned}
$$

- Rectangulo circunscrito: Es el menor rectangulo que encierra al objeto y está alineado con su orientación. A partir de ésta se rotan los puntos del contorno para buscar los valores máximos y mínimos de ambos ejes.

Los momentos invariables a rotación y referidos al sistema de coordenadas locales de la región vienen dados por:

$$
\begin{aligned}
& \mu_{i j}^{\prime}=\sum_{r=0}^{i} \sum_{s=0}^{j}(-1)^{j-s} \cdot\left(\begin{array}{l}
i \\
r
\end{array}\right) \cdot\left(\begin{array}{l}
j \\
s
\end{array}\right) \cdot(\cos \theta)^{i-r+s} . \\
& \cdot(\sin \theta)^{j+r-s} \cdot \mu_{(i+j-r-s) /(r+s)} \\
& \mu_{00}^{\prime}=\int_{-L x / 2}^{L x / 2} \int_{L y / 2}^{L y / 2} d x d y=L x \cdot L y
\end{aligned}
$$

$$
\begin{aligned}
& \mu_{02}^{\prime}=\int_{-L x / 2}^{L x / 2} \int_{L y / 2}^{L y / 2} y^{2} d x d y=\frac{1}{12} L x \cdot L y^{3} \\
& \mu_{20}^{\prime}=\int_{-L x / 2}^{L x / 2} \int_{-L y / 2}^{L y / 2} x^{2} d x d y=\frac{1}{12} L x^{3} \cdot L y
\end{aligned}
$$

Despejando $L x$ y $L y$ tendremos:

$$
L x=\sqrt{\frac{12 \cdot \mu_{20}^{\prime}}{\mu_{00}^{\prime}}} ; L y=\sqrt{\frac{12 \cdot \mu_{02}^{\prime}}{\mu_{00}^{\prime}}}
$$

Como resultado de esta fase se dispone de una aproximación bastante exacta (cuya calidad final depende de la calidad del modelo digital de superficie disponible) de las edificaciones que se utiliza como información de partida en la fase de mejora mediante el tratamiento de imágenes en la fase siguiente (Fase de Extracción) (figura 5). En el caso de no conseguir una mejora de la representación del edificio, será considerada como la representación definitiva del mismo.

\subsubsection{Fase de Extracción}

Las coordenadas terreno de estos rectángulos se transforman en coordenadas imagen (píxeles) mediante la aplicación de la condición de colinealidad (transformación de coordenadas terreno a fotocoordenadas) y una transformación afín inversa (transformación de fotocoordenadas a coordenadas imagen -píxeles-). Con estas coordenadas se obtiene el rectángulo mínimo-encuadrante para cada una de las regiones en coordenadas píxel.

A partir de este rectángulo se genera una imagen para cada región que es enviada al procedimiento general de extracción del rectángulo que representa al edificio correspondiente. A fin de reducir al máximo los tiempos de cálculo e intentar minimizar la influencia de zonas exteriores del edificio (sobre todo en edificios en los que la dirección de su planta está en la diagonal) sólo se trabaja dentro del rec- 
FIGURA 5.
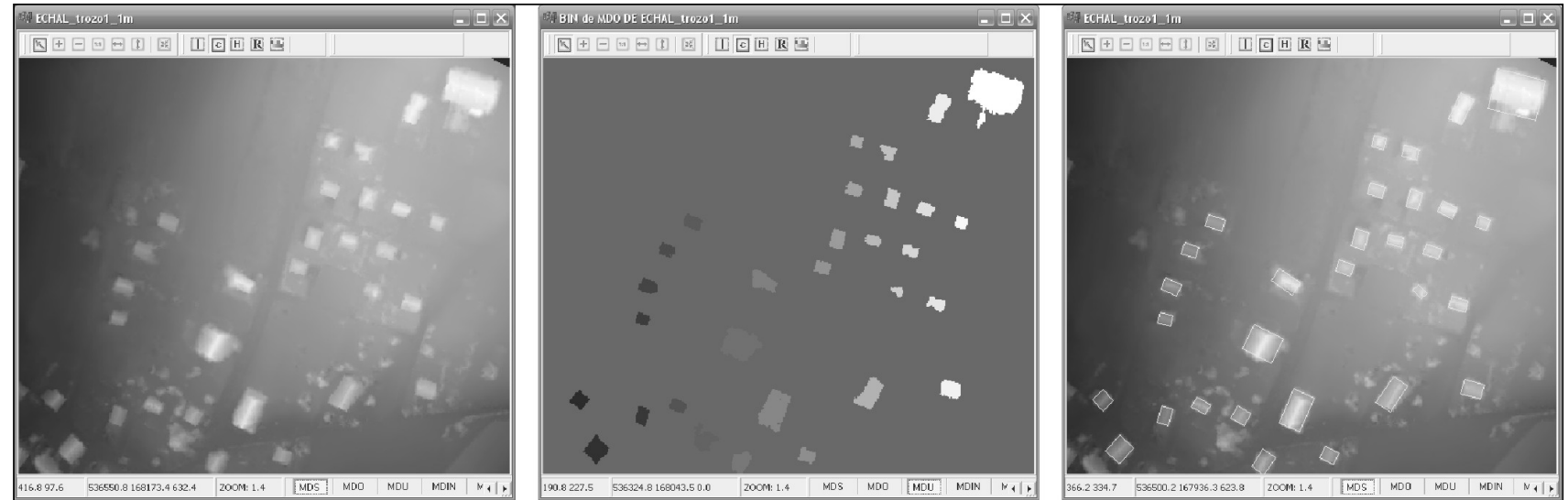

tángulo envolvente a la región considerando un cierto margen de seguridad.

El procedimiento general de extracción se resume en la figura 6, cuyas fases se explican con un mayor grado de detalle a continuación.

a) Fase de preparación o de mejora de la imagen: Si fuese necesario se aplica a la imagen una mejora del contraste mediante una expansión lineal. Esta fase que es opcional, hay que activarla para cada proyecto en función de la calidad de las imágenes.

b) Fase de extracción de bordes: Se realiza una extracción de bordes mediante la utilización del filtro de Canny (González, 2000). La imagen obtenida (imagen de bordes) es una imagen binaria donde se clasifican los píxeles en píxeles de borde y de no borde, que se utilizan en fases posteriores.

FIGURA 6.

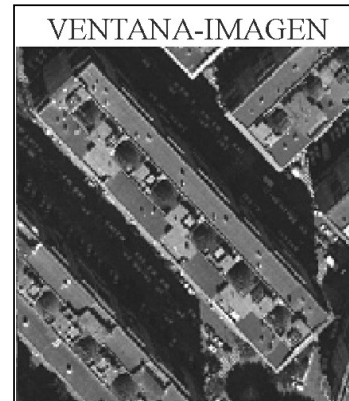

RECTAS PRINCIPALES

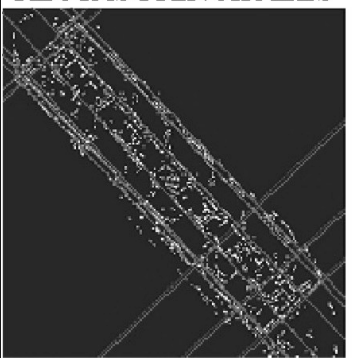

INTERSECCIONES

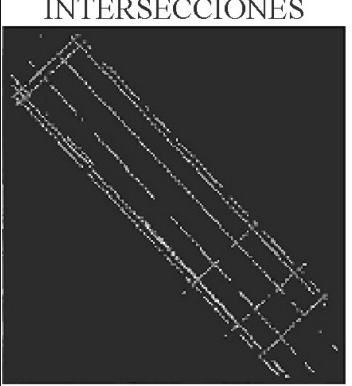

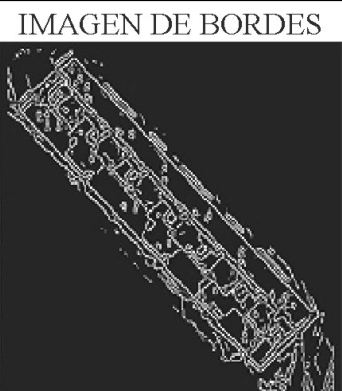

RESTAURACIÓN DE BORDES

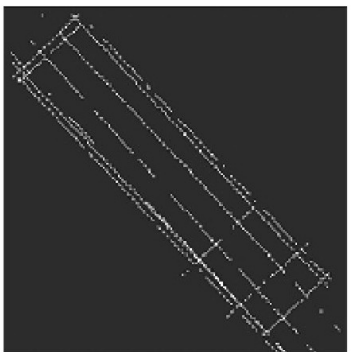

RECTÁNGULO PRINCIPAL

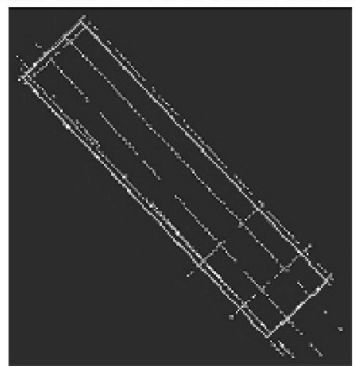

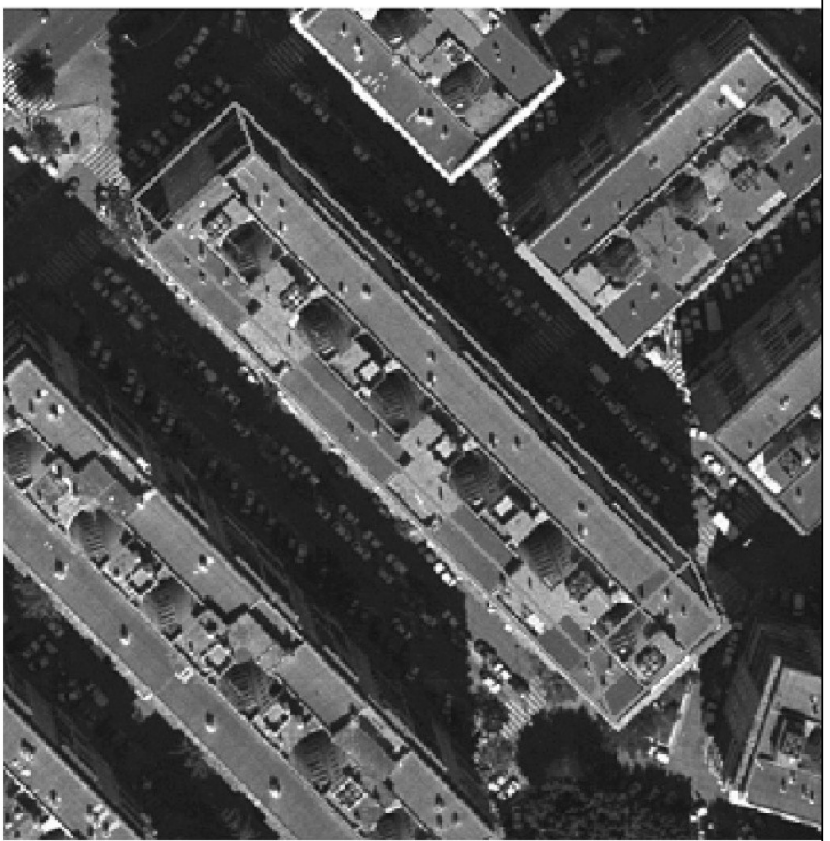

PRISMA RECTANGULAR 
c) Fase de extracción de las líneas rectas de los bordes: A continuación se aplica la transformada de Hough para líneas rectas (González, 2000) a partir de la cual se extraen las rectas de las direcciones principales de la imagen, considerando estas rectas como aquellas cuya dirección es igual o perpendicular a la dirección del gradiente principal observado en la imagen. Al trabajar de manera local y teniendo en cuenta que la imagen sobre la que se trabaja contiene de una forma casi exclusiva la superficie de la edificación extraída, la dirección del gradiente principal coincide (o es similar) con la dirección del borde principal de la imagen, que debe ser un borde del edificio.

Por otro lado es importante tener en cuenta que se parte del modelo geométrico de edificios de forma prismática (base rectangular) por lo que en la dirección perpendicular a ésta esta el otro borde del edificio. Dicho esto, una vez calculada la dirección principal del gradiente (ya calculado en la fase anterior para el filtro de Canny), se utiliza la transformada de Hough para extraer líneas rectas, pero limitándose a las direcciones antes mencionadas y a aquellas rectas cuyos contadores superen un umbral marcado.

Los valores seleccionados como óptimos tras la realización de diferentes ensayos son partir de un umbral del 30$50 \%$ del máximo obtenido en cada dirección. Es decir se extraen las rectas cuyos contadores ( $n^{\circ}$ de píxeles) estén por encima del $30-50 \%$ del contador de la recta principal de la misma dirección.

El resultado de esta fase son dos listas de rectas con pendientes perpendiculares dentro de un determinado nivel de tolerancia.

En esta fase se realiza una restauración de bordes, pero sólo de aquellos que pertenezcan a rectas principales. Para ello se utiliza la transformada de Hough inversa (González, 2000). Se restaura todo aquel píxel que no esté clasificado como borde y que perteneciendo a alguna de las rectas el gradiente tiene la dirección perpendicular a la misma y su módulo supere un segundo umbral menos exigente que el anterior.

Con estas rectas se realizan todas las intersecciones posibles entre rectas de direcciones perpendiculares, dando como resultado una lista de puntos a partir de los cuales se generan todos los rectángulos posibles teniendo en cuenta algunas limitaciones (relación entre lados, superficie del rectángulo, etc.).

A continuación se procede con la selección del rectángulo más representativo para lo cual se procede a la búsqueda de aquellos rectángulos en los que la función siguiente no supere el umbral marcado cuyas variables estarán relacionadas con los siguientes conceptos:

$$
f_{1}\left(x_{1}, x_{2}\right)<\text { umbral }
$$

$x_{1}$ : Relación entre Áreas (región y rectángulo)

$x_{2}$ : Relación de formas (lado mayor/lado menor) del rectángulo obtenido y la región correspondiente. $f_{1}=\sqrt{\left(\frac{\mid \sqrt{A_{-} \operatorname{Rec} c_{i}-\sqrt{A_{-} \operatorname{Re} g} \mid}}{\sqrt{A_{-} \operatorname{Re} g}}\right)^{2}+\left(\frac{\left|F_{-} \operatorname{Rec} c_{i}-F_{-} \operatorname{Re} g\right|}{F_{-} \operatorname{Re} g}\right)^{2}}<u$ umbra

El valor obtenido puede ser considerado como un error relativo de esta magnitud, puesto que se obtiene del cociente de la diferencia entre los datos del rectángulo y de la región y del valor del dato de la misma. La elección de este umbral debe estar en función de la calidad esperada en el rectángulo que determina la región, y de la calidad del MDS del que se disponga. El valor considerado por defecto es de 0.20 .

Esta fase permite eliminar todos aquellos rectángulos formados que se alejen de la forma y tamaño esperado, evitando la necesidad de realizar los cálculos siguientes (conteo del número de píxeles de borde en el rectángulo de la imagen bordes) en los mismos.

Por último, se seleccionan de todos los rectángulos depurados los que minimicen la función:

$$
f_{2}\left(x_{3}\right)=\min
$$

$x_{3}: N^{0}$ de píxeles de borde (Pix_Borde) en relación con el perímetro de dicho rectángulo $\left(P \_R e c\right)$

$$
\min \left(f_{2}\right)_{i} f_{2}=\frac{\mid \text { Pix_Borde }-\mathrm{P} \_ \text {Rec } 1 \text { | }}{\text { P_Rec } 1}
$$

d) Obtención del prisma rectangular: Obtenido el rectángulo más característico del edificio y considerando como altura del mismo la cota media del borde de la región correspondiente calculada anteriormente, se genera el prisma que representa al edificio (figura 6).

\subsubsection{Fase de Edición}

Puesto que se trata de una metodología semiautomática, posteriormente a la fase de extracción será necesaria una fase de edición. En esta fase se procede a la depuración de los resultados eliminando aquellos objetos considerados como edificios y que en la realidad no lo son (por ejemplo, masas arbóreas, formas especiales del relieve, trozos de edificios fracturados en la fase de apertura por la utilización de una distancia inadecuada, etc.) y editar los errores cometidos en edificios que han sido adecuadamente localizados pero cuya representación es incorrecta. Para ello el programa realiza la exportación de los resultados a un fichero formato AutoCAD DXF mediante el cual es posible realizar la edición de los resultados en un sistema fotogramétrico digital utilizando para ello la visión estereoscópica.

\section{RESULTADOS}

Para poner de manifiesto las posibilidades de la metodología propuesta a continuación se presenta un ejemplo 
completo de aplicación. La zona seleccionada está situada en la provincia de Almería y se ha partido de la siguiente información:

- Caracteristicas del modelo digital del terreno: Origen: Obtenido por correlación (fotogrametría digital).

Espaciado: $1 \mathrm{~m}$

Cota min.: $39.68 \mathrm{~m} \quad$ Cota max.: $61.85 \mathrm{~m}$

Cota med.: $45.52 \mathrm{~m}$

- Características de las tomas fotográficas.

Distancia focal: $153.33 \mathrm{~mm}$

Escala del fotograma: 1/5000

Altura de vuelo: $850 \mathrm{~m}$

Resolución de digitalización: $40 \mu \mathrm{m}$

Tamaño píxel sobre el terreno: $0.2 \mathrm{~m}$

Imagen izquierda:

$X_{0}(m)=572159.10 Y_{0}(m)=4075252.36$

$Z_{0}(m)=855.53$

$\omega($ deg $)=0.2796 \varphi($ deg $)=-0.5188 \kappa($ deg $)=2.0501$

Imagen derecha:

$X_{0}(m)=572619.53 Y_{0}(m)=4075257.82$

$Z_{0}(m)=857.82$

$\omega(\mathrm{deg})=-0.3702 \varphi(\mathrm{deg})=-0.2634 \kappa(\mathrm{deg})=2.0852$

- Caracteristicas de la escena: Zona Ilana de terreno industrial, en la que existen diversos edificios de gran tamaño y planta rectangular, que se disponen con una considerable separación entre ellos. Los edificios presentan grandes diferencias en su superficie aunque su altura es bastante uniforme. Los tejados son planos o a dos aguas, aunque también existe alguno con formas irregulares. En la figura 7 se muestra la imagen de la escena considerada.

El proceso ha realizado la extracción inicial de 18 regiones, las cuales una vez depuradas a partir de los bordes extraidos en las imágenes aéreas dan lugar a los rectángulos obtenidos mediante los momentos inerciales (línea de tono oscuro) y los edificios definitivos (línea de tono claro) que se muestran en la figura 8. Se puede observar que en ciertos casos el rectángulo oscuro no tiene edificio equivalente al no alcanzar ningún rectángulo aceptable en la fase de extracción.

En la tabla 1 se muestran los resultados numéricos obtenidos relativos a posición del edificio, superficie y altura del mismo, así como las diferencias detectadas entre los valores extraídos de forma automática y los correspondientes a la extracción mediante restitución digital empleando un sistema fotogramétrico digital con sistema de visión estereoscópica.

El primer resultado de interés es que tal y como se puede observar en la imagen de resultados la práctica totalidad de los edificios existente en la escena han sido extraídos, así como otros objetos (depósitos identificados como 1 y 4) su

FIGURA 7.

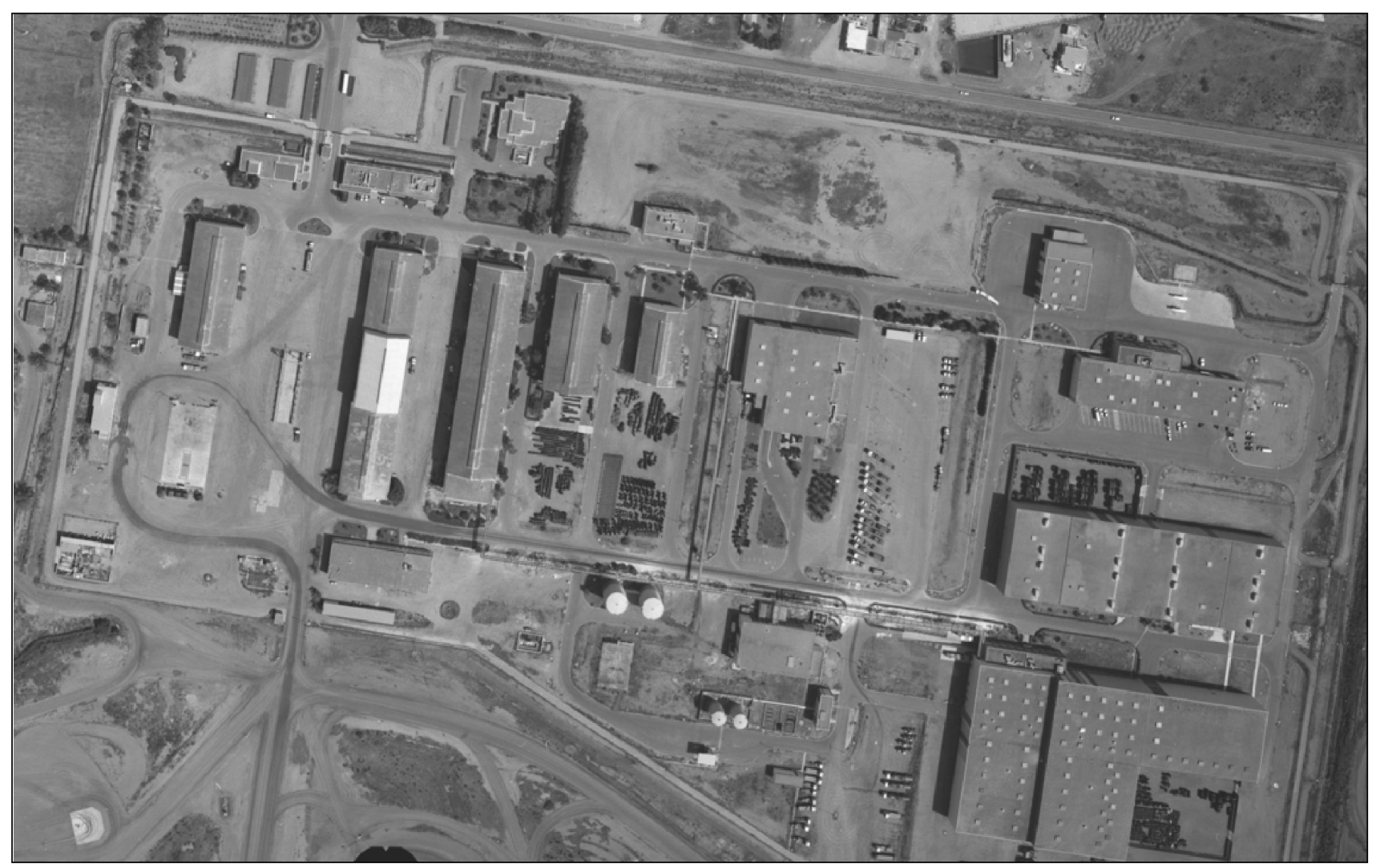


FIGURA 8.

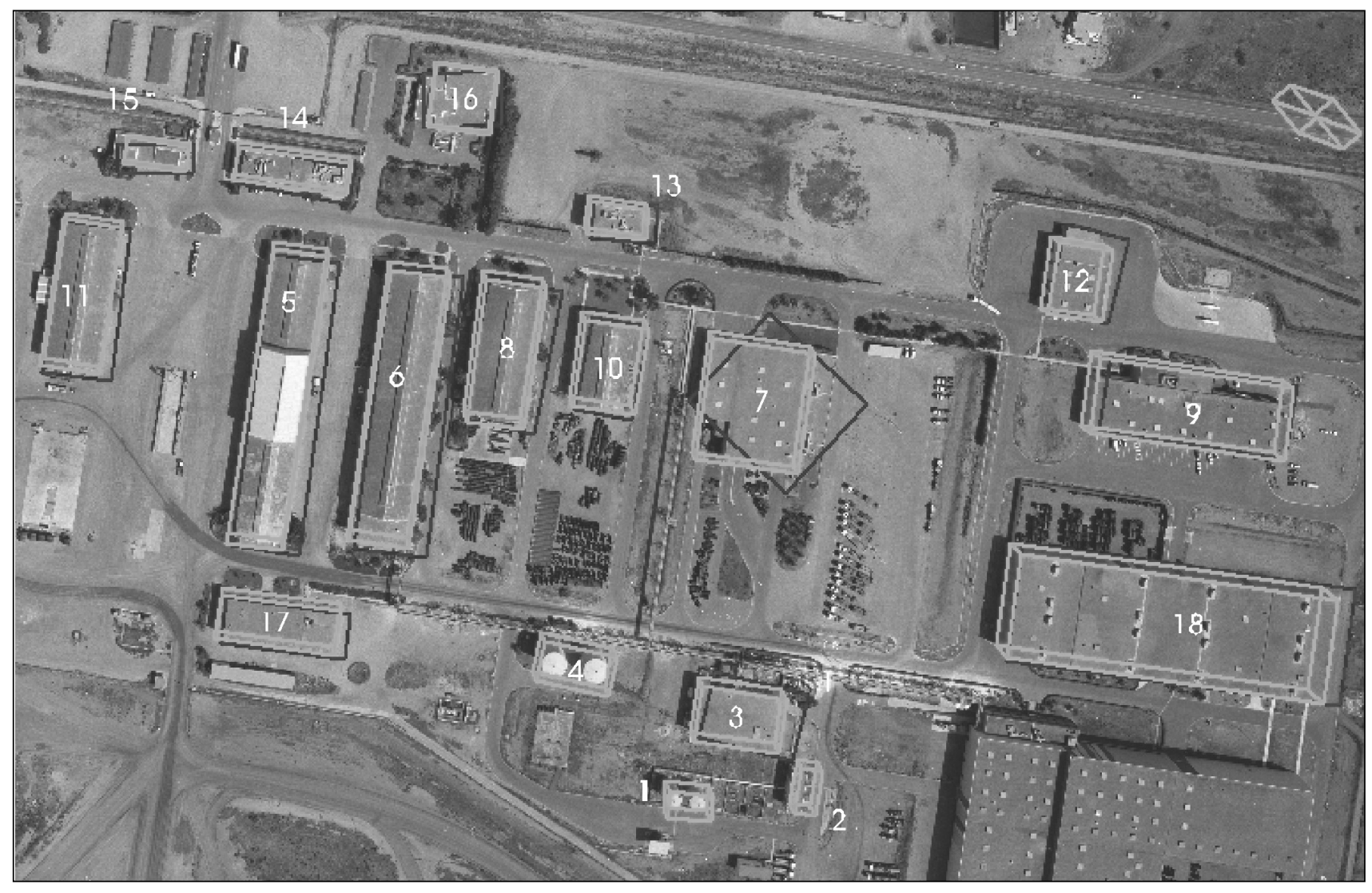

TABLA I: Resultados de la extracción

\begin{tabular}{|c|c|c|c|c|c|c|c|}
\hline & $\mathrm{Xm}$ & $\mathrm{Ym}$ & $\mathrm{Zm}$ & $\mathrm{Sup}$ & Altura & DS & $\mathrm{dh}$ \\
\hline 1 & 572376.18 & 4074759.37 & 44.93 & 261.00 & 8.13 & $\ldots$ & $\ldots$ \\
\hline 2 & 572432.36 & 4074767.26 & 47.46 & 213.00 & 7.08 & $\ldots$ & $\ldots$ \\
\hline 3 & 572398.33 & 4074799.98 & 48.07 & 1066.89 & 8.14 & -44.84 & 0.92 \\
\hline 4 & 572319.43 & 4074822.28 & 43.54 & 657.00 & 8.93 & $\ldots$ & $\ldots$ \\
\hline 5 & 572174.19 & 4074943.42 & 46.67 & 3522.52 & 11.33 & 216.30 & -3.94 \\
\hline 6 & 572231.59 & 4074941.52 & 47.67 & 3599.72 & 11.35 & -404.00 & -0.43 \\
\hline 7 & 572406.66 & 4074958.70 & 47.90 & 2299.89 & 7.80 & 832.10 & 0.74 \\
\hline 8 & 572280.98 & 4074969.34 & 47.04 & 1894.84 & 12.08 & -164.00 & -2.84 \\
\hline 9 & 572610.94 & 4074955.08 & 44.64 & 3151.00 & 8.73 & 537.70 & -0.36 \\
\hline 10 & 572331.61 & 4074963.80 & 47.31 & 1165.47 & 10.63 & -238.30 & -0.09 \\
\hline 11 & 572079.17 & 4074988.39 & 46.91 & 1948.93 & 9.82 & -68.49 & -0.17 \\
\hline 12 & 572553.08 & 4075011.59 & 48.17 & 890.87 & 8.33 & -19.19 & -0.11 \\
\hline 13 & 572332.52 & 4075032.45 & 47.01 & 430.18 & 8.02 & -58.04 & 0.45 \\
\hline 14 & 572175.53 & 4075050.67 & 45.48 & 770.34 & 8.96 & -78.44 & -1.40 \\
\hline 15 & 572115.22 & 4075054.46 & 44.70 & 303.57 & 5.40 & 129.00 & -0.62 \\
\hline 16 & 572256.73 & 4075090.35 & 45.22 & 628.57 & 6.45 & $\ldots$ & $\ldots$ \\
\hline 17 & 572177.62 & 4074836.47 & 45.35 & 1127.92 & 6.69 & -15.31 & -0.58 \\
\hline 18 & 572600.35 & 4074851.10 & 49.41 & 7426.31 & 12.82 & -150.3 & -0.48 \\
\hline
\end{tabular}

Xm, Ym, Zm: Coordenadas terreno del punto medio del edificio; Sup: Superficie el edificio; Altura: Altura máxima del edificio; DS: Diferencia de superficie; dh: Diferencia de altura (Todos los valores en $\mathrm{m}$ )

80 Uned. Espacio, Tiempo y Forma. Serie VI, Nueva época. Geografía, n. 1, 2008. 
posición detectada y su representación refinada mediante el análisis de sus bordes.

Con respecto al análisis de las diferencias la consideración de estos valores debe tener en cuenta dos aspectos fundamentales: en primer lugar, el hecho de que el MDS considerado ha sido de tipo integer por lo que las cotas se han aproximado al entero más próximo; en segundo lugar; que la cota media del edificio hace referencia a la cota media del rectángulo en el que se sitúa el edificio lo que tiene influencia especialmente en los tejados a dos aguas en los que los valores medios de cota son de complejo cálculo a partir de los resultados de la restitución estereoscópica. No obstante, es importante indicar que los errores relativos medios obtenidos son del orden del $1 \%$ en superficie y de un $5 \%$ en la altura de los mismos, por lo que se puede indicar que la representación de la escena es aceptable y realista.

\section{CONCLUSIONES}

Los resultados obtenidos en este trabajo ponen de manifiesto la utilidad tanto de la metodología planteada como de la aplicación informática desarrollada para la extracción semiautomática de edificios siempre que éstos se ajusten al modelo prismático planteado en la metodología. La información de partida del sistema es básicamente las imágenes monoscópicas orientadas de la zona así como un MDS de la zona considerada (que puede ser obtenido fácilmente mediante correlación utilizando sistemas fotogramétricos digitales o mediante sistemas LIDAR).

Tal y como se ha señalado en el apartado introductorio, la extracción de la información que permitan una descripción tridimensional de los edificios a partir de las imágenes y el MDS de la zona no es una tarea sencilla. Es importante que el sistema sea capaz de discriminar entre la información "útil» y la que enmascara y "ensucia" a la anterior, y por otro lado, el enorme volumen de información que es necesario considerar (imágenes digitales fotogramétricas) implica que es necesario restringir la zona de trabajo a aquellas áreas en las que existan los edificios, lo que se consigue mediante la información adicional proporcionada por el MDS.

La extracción del rectángulo más idóneo depende en gran parte de la calidad de la región obtenida en la fase de localización, por lo que una cuestión a tener en cuenta es que el modelo aportado a la aplicación ha de ser preciso y que describa fielmente la escena a estudiar. En caso contrario partiremos de zonas de búsqueda erróneas y de características de edificios (necesarias para la depuración de la información extraída mediante el análisis de las imágenes) falsas, lo que nos podría llevar a realizar una depuración errónea despreciando el rectángulo correcto. En el caso de modelos obtenidos mediante correlación (caso general en fotogrametría digital) los modelos obtenidos no se adaptan al terreno demasiado bien en zonas de edificaciones altas, de- bido sobre todo a la presencia de zonas ocultas y que a cambios bruscos de cota equivalen valores grandes de paralaje. Un aspecto importante a destacar de la metodología propuesta es que permite la utilización de MDS de diferente fuente, como por ejemplo, LIDAR con lo que estos problemas están solventados.

Otra ventaja importante es el trabajo a escala local, ya que a pesar de que la localización de las regiones haya sido correcta, siempre van a aparecer dificultades para seleccionar de entre todos los rectángulos posibles, el que realmente se ajusta al edificio. Así en la mayoría de los casos, la existencia de estructuras en los tejados como chimeneas, terrazas, patios interiores, etc., la aparición de paredes donde se muestran elementos tales como ventanas, bordillos, y otros objetos, además de las sombras proyectadas por los edificios, se van a disponer de forma paralela a los bordes de los tejados de los mismos. Esto introduce un nivel de ruido elevado que dificulta la selección de los segmentos lineales que determinarán posteriormente el rectángulo definitivo.

\section{AGRADECIMIENTOS}

Este trabajo ha sido realizado en la Universidad de Jaén en el marco del proyecto "INARA.CARTO: Desarrollo de metodologias e instrumentos (software y hardware) para la explotación optima de sistemas mixtas de sensores LIDAR y cámaras digitales». El proyecto es liderado por la empresa Stereocarto S.L. y financiado por el Plan Nacional de Investigación Científica, Desarrollo e Innovación Tecnológica (FIT-3501002005-462) del Ministerio de Industria, Comercio y Turismo, el Plan de Innovación Empresarial de la Comunidad Autónoma de Madrid (PIE/1043/2005) (IMADE) y el Centro de Desarrollo Tecnológico Industrial (CDTI).

Este trabajo forma parte de las actividades del grupo de investigación "Sistemas Fotogramétricos y Topométricos» del Plan Andaluz de Investigación dependiente de la Consejería de Innovación, Ciencia y Empresa de la Junta de Andalucia, parcialmente financiado con fondos FEDER de la Unión Europea.

\section{BIBLIOGRAFÍA}

BaltSAVIAS, E.; MASON, S. y Stallman, D. (1995): "Use os DTMs/DSMs and Orthoimages to Support Building Extraction» En: Gruen, A.; Kuebler, 0. y Augouris, P. (Ed.): Automatic Extraction of Man-Made Objets from Aerial and Space Images. Monte Veritá, Birkhäuser.

Gerke, M.; HeIPKE, C. y Straub, B. M. (2001): "Building Extraction From Aerial Imagery Using a Generic Scene Model and Invariant Geometric Moments". Proceedings of the IEEE/ISPRS joint Workshop on Remote Sensing and Data Fusion over Urban Areas, November 89th 2001, University of Pavia, Rome (Italy), pp. 85-89.

GonZÁlEZ, J. (2000): «Visión por computadon.Editorial Paraninfo, Madrid.

GonZÁlEZ, R.C. y Woods, R.E. (1996). "Tratamiento Digital de Imágenes» Addison-Wesley lberoamericana S.A., Madrid. 
José Luis Pérez Garcia y Jorge Delgado García

Lin, C.; Huertas, A. y Nevatia, R. (1995): «Detection of Building from Monocular Images» En: Gruen, A.; Kuebler, O. y Augouris, P. (Ed.): Automatic Extraction of Man-Made Objets from Aerial and Space Images. Monte Veritá, Birkhäuser.

LIN, C. y NevatiA, R. (1998): «Building Detection and Description from a Single Intensity Imagem. Computer Vision and Image Understanding, 72, 2, pp. 101-121.

Sithole G. (2001). "Filtering of laser altimetry data using a slope adaptive filtern. International Archives of Photogrammetry and Remote Sensing,Volume XXXIV-3/W4

Vosselman, G. (2002): «Fusion of Laser Scanning Data, Maps, and Aerial Photographs for Building Reconstruction». Photogrammetry and
Remote Sensing Section, Department of Geodesy, Delf University of Technology.

WeIDNER, U. y FörSTNER, W. (1995): «Towards Automatic Building Extraction from High Resolution Digital Elevation Models». ISPRS Journal of Photogrammetry \& Remote Sensing, 50, 4, 38-49.

ZhANG, K.; Chen, S.C.; WhitMAn, D.; SHYU, M.L.; YAN, J. y ZHANG, C. (2003): "A Progressive Morphological Filter for Removing Nonground Measurements From Airborne LIDAR Datan. IEEE Transactions on Geoscience and Remote Sensing, 41, no. 4. 